\title{
Dual fibular allograft dowel technique for sacroiliac joint arthrodesis
}

\author{
Authors Robert A McGuire' ${ }^{1}$,Zenggan Chen ${ }^{2}$, Kevin Donahoe ${ }^{1}$ \\ Institutions ${ }^{1}$ Department of Orthopedic Surgery and Rehabilitation, University of Mississippi Medical Center, \\ Jackson, MS, USA \\ ${ }^{2}$ Department of Orthopedic Surgery, Fudan University, Shanghai, China
}

\begin{tabular}{l|l|}
\hline Final class of & Yes \\
evidence-treatment & \\
\hline Study design: \\
\hline RCT \\
Cohort \\
Case control \\
\hline Case series \\
\hline Methods \\
\hline Concealed allocation (RCT) \\
\hline Intention to treat (RCT) \\
\hline Blinded/independent \\
evaluation of primary outcome & \\
\hline F/U $\geq 85 \%$ & \\
\hline Adequate sample size & \\
\hline Control for confounding & \\
\hline Overall class of evidence & IV \\
\hline
\end{tabular}

\section{ABSTRACT}

Study design: Retrospective case series.

Objective: To assess fusion rates in patients with sacroiliac joint (SIJ) pain following a minimally invasive technique using fibular dowel allograft.

Methods: Thirty-seven consecutive patients (mean age: 42.5 years [range, 23-63 years]) with SIJ pain treated with 38 minimally invasive elective SIJ arthrodeses were retrospectively reviewed using chart and $\mathrm{x}$-ray data. The fusion procedure consisted of minimal muscle stripping over the posterior SIJ and insertion of a cranial and caudal fibular dowel graft across the joint following placement of Steinmann pins. Fusion was deemed to be present when bone bridging trabeculae could be seen crossing the SIJ on either oblique $\mathrm{x}$-rays or by computed tomographic scan. Patients were followedup for a mean of 52 months (range, 24-62 months). Visual Analog Scale (VAS) was used to monitor clinical pain improvement.

Results: Thirty-four patients with SIJ arthrodeses (89.5\%) healed and led to substantial improvement in VAS pain scores (preoperative 9.1, postoperative 3.4) $(P<.001)$. This improvement in VAS occurred over a 6-month period and was sustained through subsequent follow-up. Nonunion occurred in four patients with SIJ $(10.5 \%)$. Each SIJ nonunion was successfully treated by secondary autogenous bone grafting and compression screw fixation.

Conclusions: In patients with primary low back pain attributable to the SIJ, a minimally invasive, dual fibular dowel graft provided high rates of fusion and improved pain scores. 


\section{STUDY RATIONALE AND CONTEXT}

Although early and/or mild sacroiliac joint (SIJ) disease may be treated nonoperatively, severe or unresponsive SIJ disease may require surgical intervention. Fibular allografts may obviate the need for a separate bone donor site and provide sufficient structural support so that additional internal fixation may not be needed. Minimal muscle stripping over the posterior SIJ may provide sufficient exposure of the posterior SIJ for reliable arthrodesis while reducing the morbidity that might occur from a more extensive exposure.

\section{OBJECTIVE}

To assess fusion rates following a minimally invasive technique using fibular dowel allograft in patients with SIJ pain as an only or major contributing source of primary low back pain.
Fig 1 Patient sampling and selection. SIJ indicates sacroiliac joint.

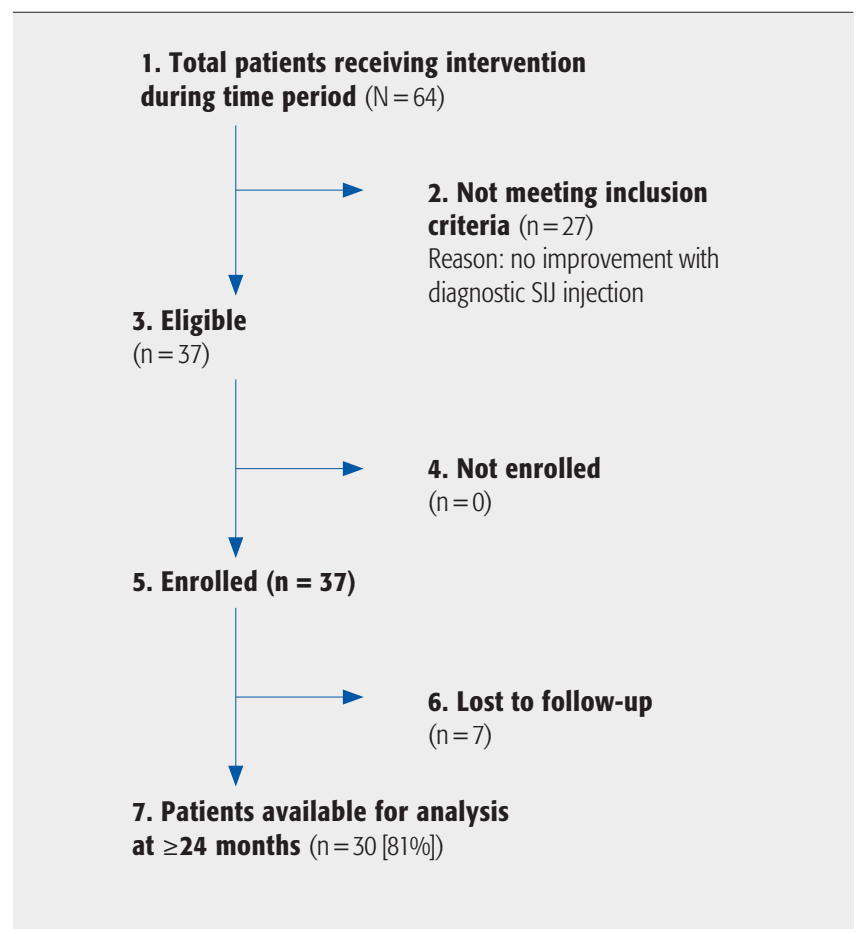

\section{METHODS}

Study design: Retrospective case series.

Inclusion criteria: Patients with SIJ pain unrelieved with conservative treatment were included. However, they obtained substantial pain relief with diagnostic SIJ injections for expected anesthetic agent time frame using two separate computed tomographic (CT)-directed injections with long- and short-duration anesthetic. Sixty-four patients were consecutively evaluated whose pain was believed to be SIJ related.

Exclusion criteria: Patients not obtaining relief from diagnostic blocks were excluded. Twenty-seven patients with SIJ pain were reviewed but were excluded due to inadequate diagnostic block-pain relief.

Patient population (Fig 1): Thirty-seven consecutive patients with SIJ pain treated between 1985 and 2006 with 38 minimally invasive elective SIJ arthrodeses were retrospectively reviewed using chart and x-ray data. Patients were followed-up an average of 39.6 months (range, 8-62 months). Thirty-four (92\%) of 37 patients were evaluated at 12 months and $30(81 \%)$ of 37 were followed-up for more than 2 years. Seven patients were lost to follow-up at 2 years-two died and five moved to an unknown location.

\section{Intervention:}

- A curvilinear incision was made over the posterosuperior iliac spine (PSIS) adjacent to the implicated SIJ (Fig 2).

- The periosteum and the fascia of the gluteus maximus overlying the PSIS was incised, elevated, and reflected to either side leaving good flaps for closure (Fig 3).

Fig 2 A standard posterior curvilinear skin incision determined by intraoperative image intensification visualization of the sacroiliac joint.

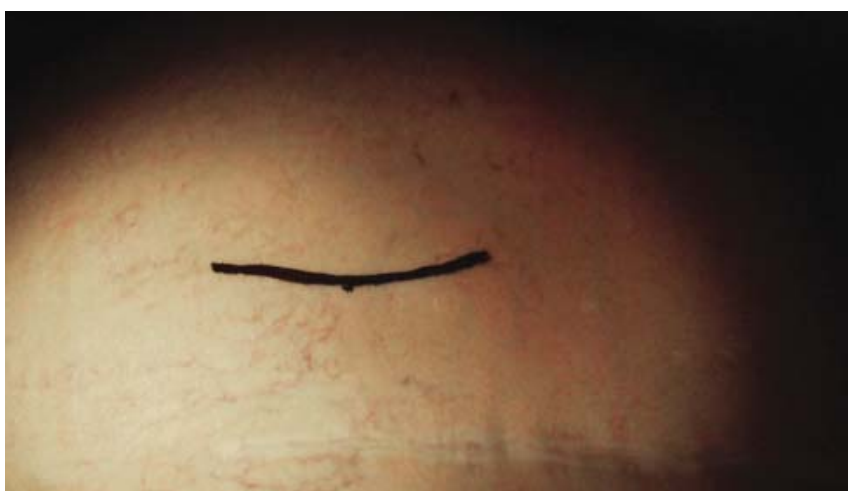


- The PSIS was removed with an osteotome to expose the SIJ (Fig 4).

- Two $3.2 \mathrm{~mm}$ Steinmann guide pins were placed into the SIJ (one cephalad and one caudal) and appropriate placement confirmed with intraoperative image intensification (Fig 5).

Fig 3 (a) Exposure of the posterior superior iliac spine (PSIS) and (b) a similar visualization of the PSIS exposure on a model.
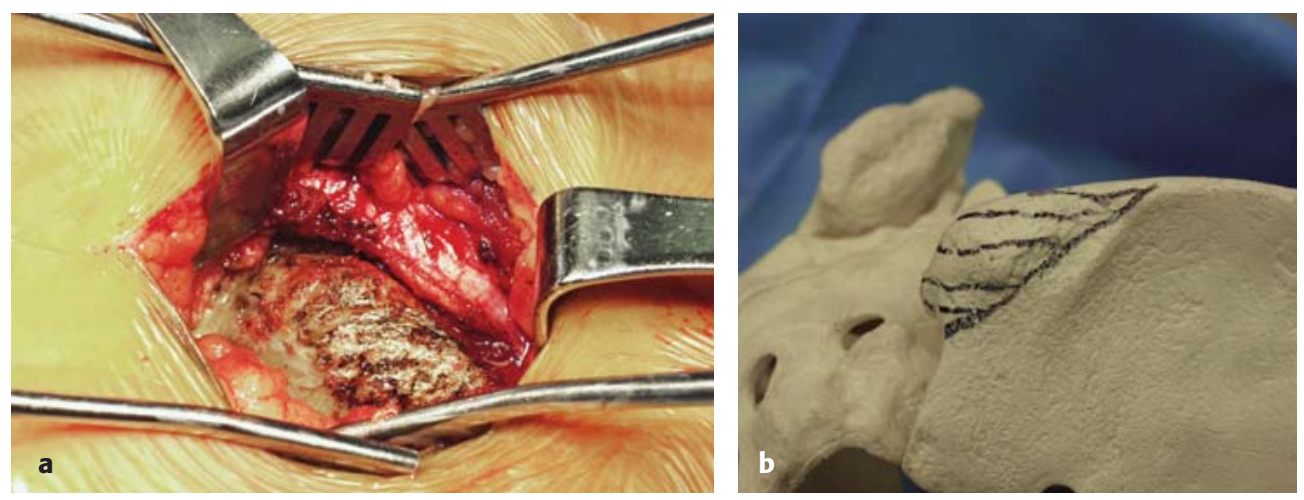

Fig 4 (a) Removing a portion of the posterior superior iliac spine to allow placement of a Steinmann pin into the sacroiliac joint. (b) Placement of the osteotome using model.
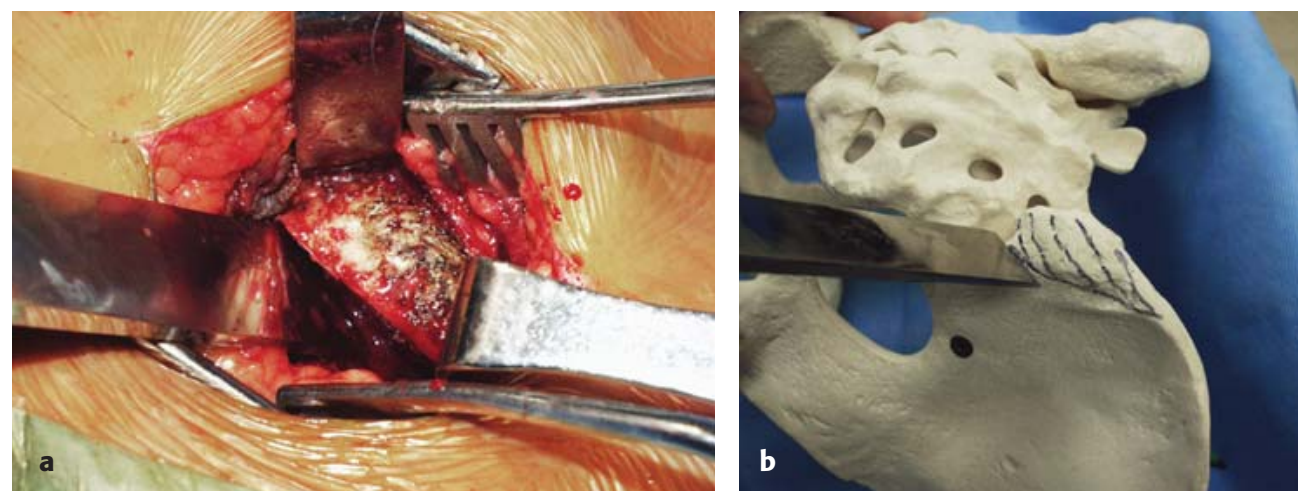

Fig 5 (a) Placement of Steinmann pins into sacroiliac joint using image intensifier guidance. (b) Position on model of Steinmann pin placement.
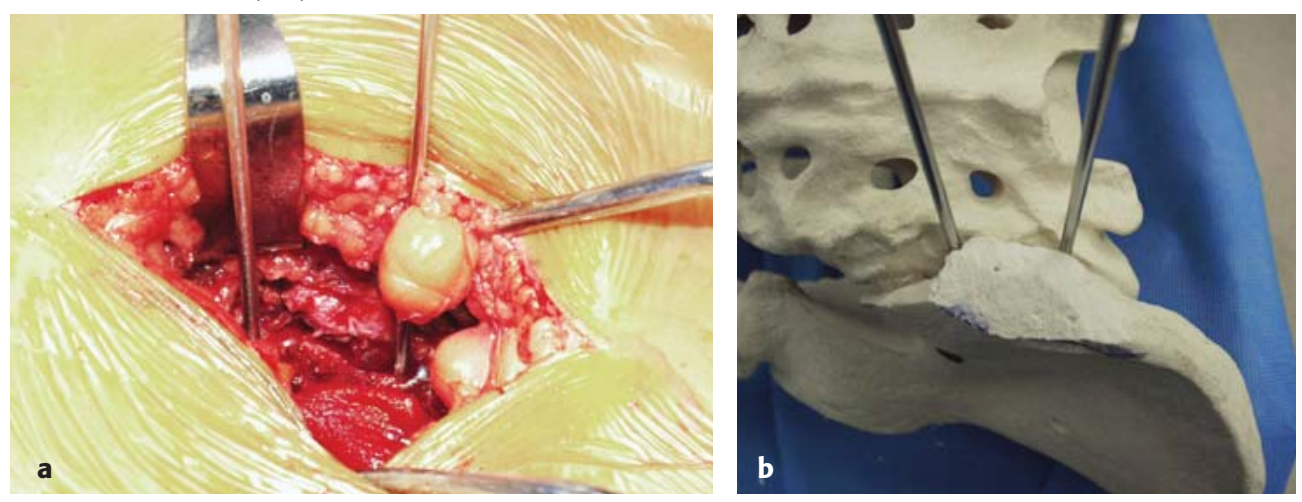


\section{Intervention (cont):}

- The fibular allograft was cut into two plugs that corresponded to the lengths of the inserted portions of the two guide pins (Fig 6).

- An anterior cruciate ligament reamer slightly smaller than the diameter of the allograft (usually 10-12 mm) was marked so that it could be drilled to a depth for the cephalad dowel hole, as predetermined from the preoperative CT scan (Fig 7).

- The SIJ was then reamed over the cephalad guide wire, removing the articular cartilage and subchondral bone on both the iliac and sacral sides of the joint (Fig 8).
- The caudal dowel hole was then reamed over the guide wire to an equal depth (Fig 9).

- Autograft was then packed into the bottom of the hole and the caudal fibular allograft inserted, rotated to impart SIJ distraction, and seated (Fig 10).

- Intraoperative image intensifier was used to confirm appropriate placement of both fibular allografts (Fig 11).

- The remainder of the morselized posterior iliac crest bone graft was packed into the SIJ and around the fibular allografts (Fig 12).

Additional detail of techniques used can be found in the Web Appendix at www.aospine.org/ebsj
Fig 6 Fibular allograft cut to appropriate lengths slightly greater in diameter than the anterior cruciate ligament reamer.

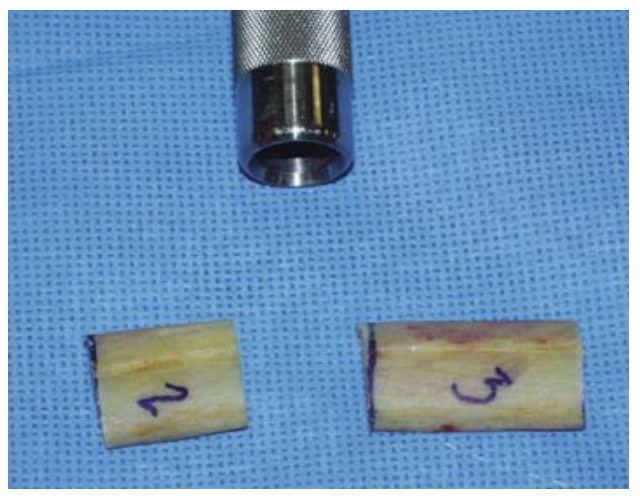

Fig 7 Setting the depth of the anterior cruciate ligament reamer to prevent breach of the anterior portion of the sacroiliac joint.

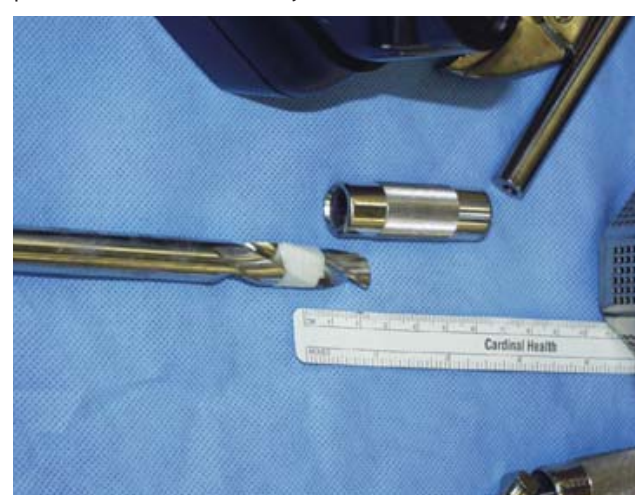

Fig 8 (a) Placement of the anterior cruciate ligament reamer over the caudal Steinmann pin that has been placed under image intensifier guidance into the sacroiliac joint between the ileum and sacrum to allow reaming of a portion of bone from each side of the joint. (b) A similar view of the anterior cruciate ligament reamer over the caudal Steinmann pin on the model.

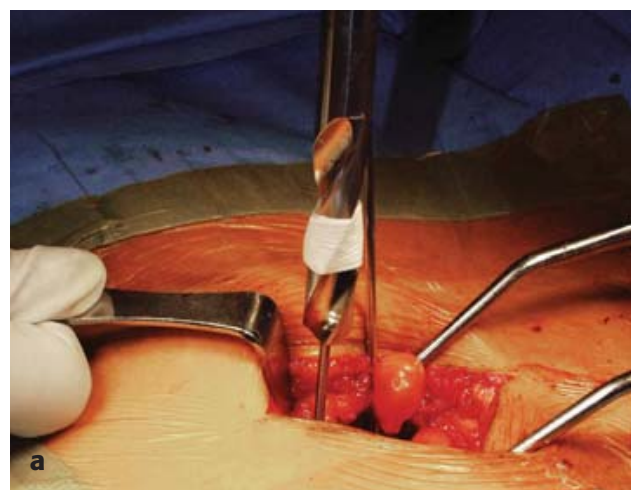

Fig 9 On the model, the appropriate reaming of the sacroiliac joint so that a portion of bone from the ileum and sacrum is removed.

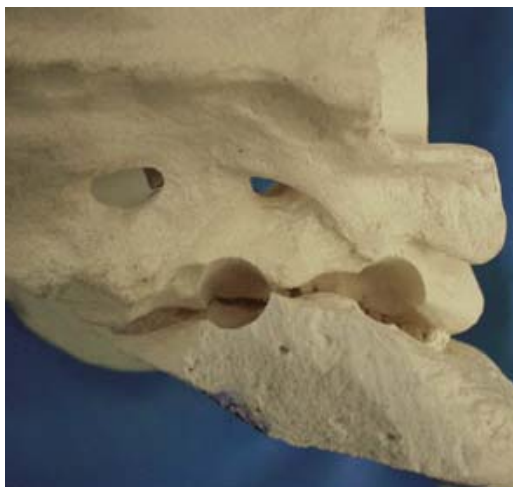




\section{Outcomes:}

- Patients were followed-up at 3 months, 6 months, 1 year, and yearly thereafter with x-rays and CT scans as needed.

- Fusion was deemed to be present when bonebridging trabeculae could be seen crossing the SIJ on either oblique x-rays or by CT scan. Early bone bridging could be seen at 3 months with improvement in pain scores noted. Oblique $x$-rays at 6 months revealed 26 of 38 SIJ fusions had good bone bridging the joint. Findings that were doubtful on oblique $\mathrm{x}$-rays or those who had increasing pain were evaluated with CT scan. Four of these patients were noted to have a nonunion and eight had evidence of fusion.

- Nonunion was defined as failure to see bridging trabeculae across the joint by 24 weeks.
- Pseudarthrosis was believed to be present if lytic lines were seen between the graft and either the ilium or sacrum.

- Visual Analog Scale (VAS) on a 10-point scale was used to assess pain at baseline, 3 months, 6 months, 12 months, and yearly thereafter. A change of 4 points in VAS from preoperative to postoperative was used as the minimal clinical difference in determining pain improvement with this procedure [1-3].

Analysis: Paired $t$ test was used to analyze data with a decrease in 4 points on a 10 -point VAS being noted to be clinically significant in this patient population (Table 1). Fusion rate was calculated as a ratio of those joints noted to have bone bridging the joint either on oblique x-rays or CT scan to the total of those surgically treated.
Fig 10 (a) Placement of the fibular allograft into the reamed space within the sacroiliac joint. (b) Similar photo of fibula allograft placement in the model.

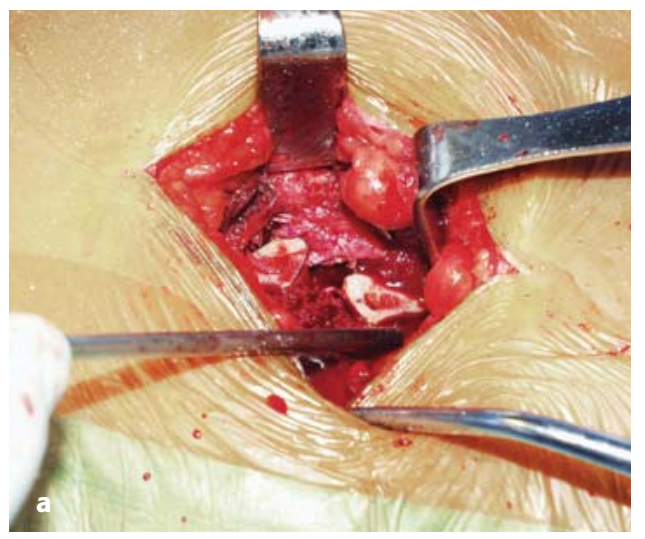

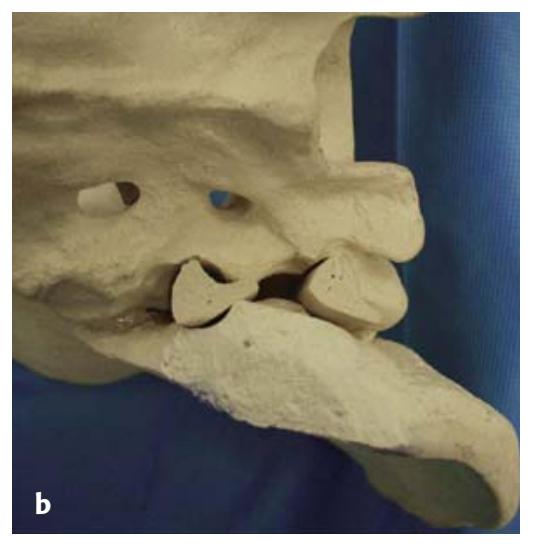

Fig 12 (a) Before wound closure, morselized bone from the portion of the posterior superior iliac spine that was removed is placed across the sacroiliac joint. (b) Using the model, placement of the morselized posterior superior iliac spine is noted.
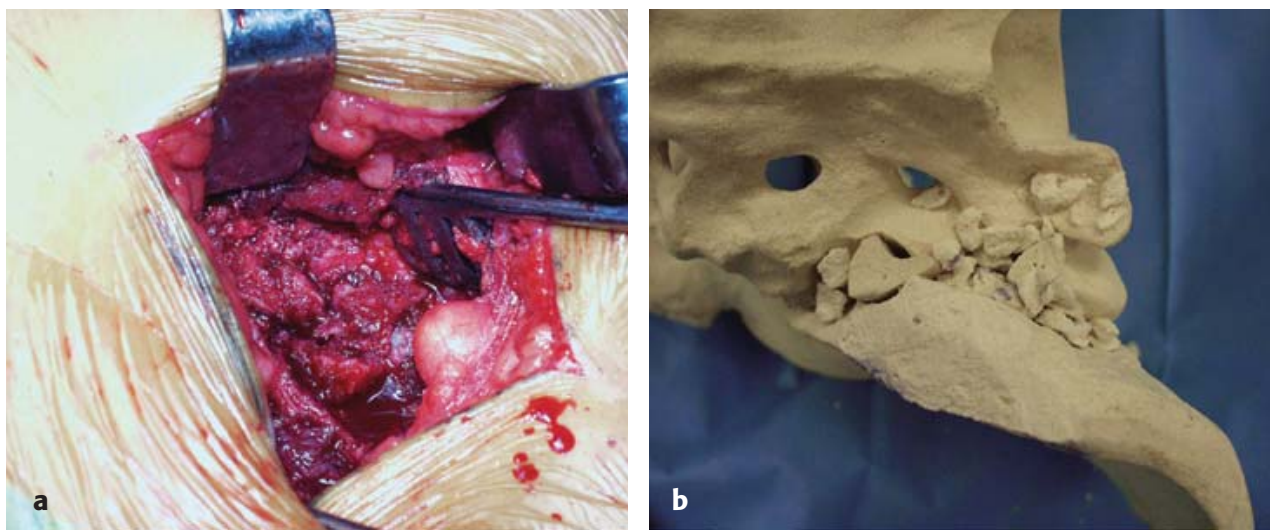

Fig 11 Intraoperative image intensifier view confirming the appropriate placement of the fibular allograft.

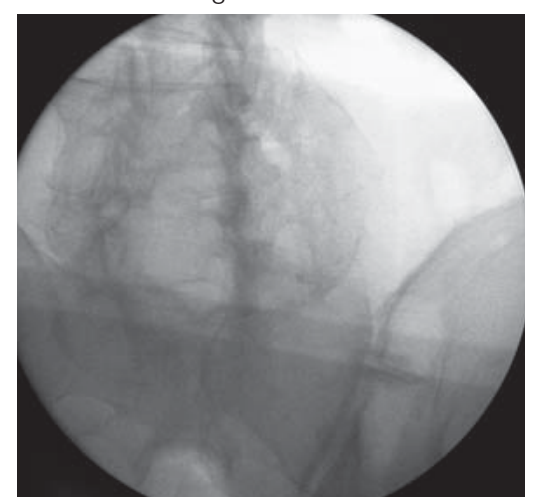


Table 1 Demographic and baseline characteristics of intervention groups.

\begin{tabular}{ll}
\hline & Treatment $(\mathbf{n = 3 7 )}$ \\
\hline Mean age, $y$ (range) & $42.5(23-63)$ \\
\hline $\begin{array}{l}\text { No. }(\%) \\
\text { Female }\end{array}$ & $34(92)$ \\
\hline Male & $3(8)$ \\
\hline Baseline Visual Analog Scale & 9.1 \\
\hline $\begin{array}{l}\text { Patients follow-up } \\
\text { Mean (range), mo }\end{array}$ & $39.6(8-62)$ \\
\hline At least 12 mo & $34(92 \%)$ \\
\hline More than 24 mo & $30(81 \%)$ \\
\hline
\end{tabular}

Table 2 Outcomes being studied.

\begin{tabular}{ll}
\hline Outcome & SIJ fusion, $\mathbf{n = 3 8}$ \\
\hline Visual Analog Scale postoperative, final & $\begin{array}{l}3.4 \text { (94\% achieved clinical } \\
\text { important difference at } \\
6 \text { mo and maintained } \\
\text { through follow-up) }\end{array}$ \\
\hline No. (\%) & $34(89.5)$ \\
\hline Fusion & $4(10.5)$ \\
\hline Nonunion & \\
\hline
\end{tabular}

\section{RESULTS}

- Thirty-seven patients, ie, 3 men and 34 women, ranging in age from 23-63 years who underwent 38 SIJ fusions were studied.

- $\quad$ Thirty-four primary SIJ arthrodeses (89.5\%) healed and led to substantial improvement or resolution of symptoms, as noted in VAS.

- Nonunion occurred at four sites (10.5\%). Each SIJ nonunion was successfully treated by secondary autogenous bone grafting and iliosacral compression screw fixation. There were no infections.

- A decrease in VAS was seen from 9.1 preoperatively to 3.4 postoperatively at final follow-up $(P<.001)$ and $35(94 \%)$ of 37 patients achieved improved VAS of 4 points (Table 2 ).

- This improvement was reached at 6 months postoperatively and was maintained for the remainder of follow-up period.

\section{DISCUSSION}

- Our study using a dual fibular dowel technique provides fusion rates $(89.5 \%)$ that compare favorably with those studies [4-8] where more invasive fusion techniques were used.

- Our data suggests that a minimally invasive approach and dual fibular allograft "interference-fit" arthrodesis can safely achieve acceptable healing rates and pain improvement in patients whose pain is linked to SIJ.

- Fibular allografts obviated the need for a separate bone donor site and provided sufficient structural support so that additional internal fixation was not initially necessary.

- Strengths of this case series include evidence of improved pain scores with this technique as well as acceptable arthrodeses rates.

- Limitations of this study include lack of direct comparison with other described techniques and a direct comparison with a similar cohort of nonoperatively treated patients with SIJ pain.

- The potential clinical impact of this study is the application of a minimally invasive surgical technique that achieves acceptable fusion rates with improvement in pain scores in patients with SIJ pain who fail conservative management.

- To establish the benefits of the dual fibular graft, a prospective study comparing it with other minimally invasive techniques using porous metal implants directed laterally across the SIJ will allow direct evaluation of the potential equality or superiority of this promising new technique.

\section{SUMMARY AND CONCLUSIONS}

- Fibular dowel allografts appear to facilitate high rates of SIJ fusion in patients with primarily low back pain of sacroiliac origin.

- Minimal muscle stripping over the posterior SIJ was a strategic component of our operative technique. Our goal was to gain sufficient exposure of the posterior SIJ for reliable arthrodesis while reducing the morbidity that might occur from a more extensive procedure.

- Dual fibular dowel SIJ fusion has been shown to decrease pain scores in patients with pain attributed to SIJ. 


\section{ILLUSTRATIVE CASES}

\section{Case 1}

Sacroiliac joint dysfunction below lumbar fusion to the sacrum (adjacent segment disease) (Fig 13): A 63-year-old man had previous lumbar fusion to the sacrum and developed increasing pain in the sacroiliac region. X-ray studies revealed degenerative changes in SIJ (adjacent segment disease). Conservative treatment measures failed and after diagnostic blocks verified SIJ as a pain generator, he underwent sacroiliac fusion with the minimally invasive SIJ dowel technique. This procedure led to sustained pain relief 1 year after surgery (VAS of 10 preoperatively to VAS of 1 postoperatively).

\section{Case 2}

A 29-year-old woman had a 2-year history of SIJ pain after falling directly on her buttocks (Fig 14). Nonoperative treatment failed to relieve her pain. Physical examination was consistent with pain of SIJ origin. Diagnostic blocks relieved the pain. The patient was treated with minimally invasive SIJ dowel arthrodesis. Six years later, she remains asymptomatic and $\mathrm{x}$-rays revealed complete SIJ arthrodesis (VAS of 9 preoperatively to VAS of 0 postoperatively).

Fig 13 Postoperative oblique $x$-ray revealing excellent bone healing across the sacroiliac joint.

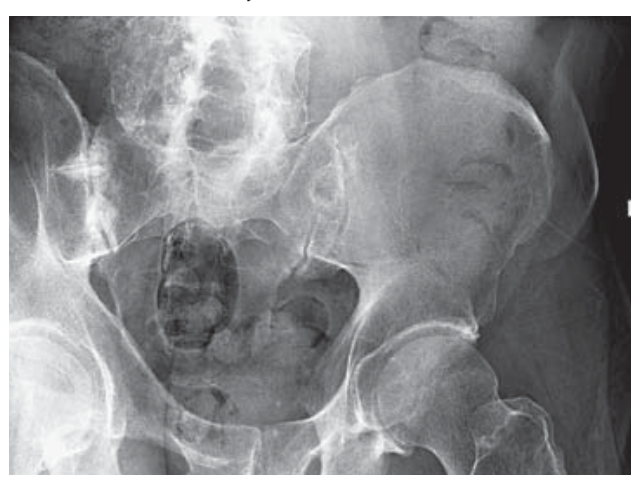

Fig 14 Complete obliteration of the sacroiliac joint is noted on this oblique $x$-ray evaluation of the joint.

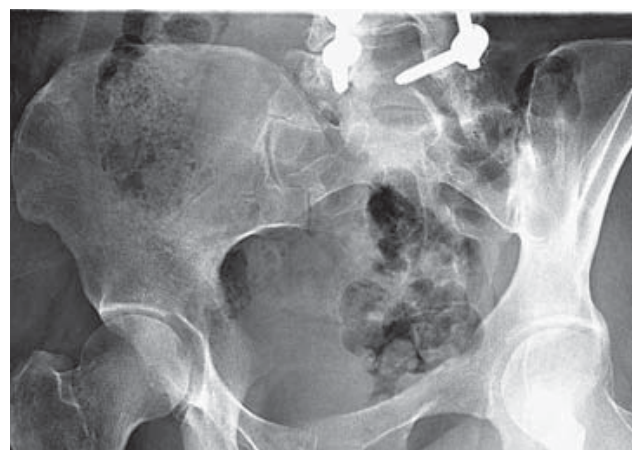

\section{REFERENCES}

1. Salaff F, Stancati A, Silvestri CA, et al (2004) Minimal clinically important changes in chronic musculoskeletal pain intensity measured on a numerical rating scale. Eur J Pain; 8(4):283291.

2. Ostelo RW, Devo RA, Stratford P, et al (2008) Interpreting change scores for pain and functional status in low back pain: towards international consensus regarding minimial important change. Spine (Phila Pa 1976); 33(1):90-94.

3. Ostelo RW, deVet HC (2005) Clinically important outcomes in low back pain. Best Pract Res Clin Rheumatol; 19(4):593-607.

4. Routt MLC Jr, Kregor PJ, Simonian PT, et al (1995) Early results of percutaneous iliosacral screws placed with the patient in a supine position. J Orthop Trauma; 9(3):207-214.

5. Tonetti J, Carrat L, Lavalleé S, et al (1998) Percutaneous iliosacral screw placement using image guided techniques. Clin Orthop Relat Res; (354):103-110.

6. van den Bosch EW, van Zwienen $\mathrm{CM}$, van Vugt AB (2002) Fluoroscopic positioning of sacroiliac screws in 88 patients. J Trauma; 53(1):44-48.

7. Buchowski JM, Kebaish KM, Sinkov V, et al (2005) Functional and radiographic outcomes of sacroiliac arthrodesis for the disorders of the sacroiliac joint. Spine J; 5(5):520-528; discussion 529.

8. Belanger TA, Dall BE (2001) Sacroiliac arthrodesis using a posterior midline fascial splitting approach and pedicle screw instrumentation: a new technique. J Spinal Disord; 14(2):118-124. 


\section{EDITORIAL PERSPECTIVE}

The topic of sacroiliac joint (SIJ) pain and its management remains one filled with controversy and doubt. As previously discussed in an EBSJ systematic review titled, "Chronic sacroiliac joint pain: fusion versus denervation as treatment options" [1], the very diagnosis of a symptomatic SIJ remains uncertain - with no specific tests having been established over others as being definitive, treatment of the painful SIJ becomes all the more an intuitive application [2].

McGuire and colleagues describe a sensible approach to diagnostic testing. Of all injection techniques, a CT-based approach, as described in the article, remains the most unequivocal way to assess pain relief imaginable to date.

As to the surgical techniques for SIJ fusions, this again is a highly controversial area of spine surgery and trauma orthopaedics since there is not one technique that has clearly been established to combine safety of application with good and lasting outcomes in a predictable fashion. There has been renewed interest by the industry in 'discovering' SIJ as a source of low back pain (and with that as a potential business model).

Not surprisingly, a number of new products and techniques has been or will be introduced in the near future to address this perceived 'need.' This technique is different as it does not attempt to cross the SJI but rather provides an interference fit within the main excursion of the joint. This promises to be a safer and less complex undertaking than either crossing the SI joint or placing hardware through an anterior approach. At this point in time we have a single-surgeon case series, with reasonable results.

1. Ashman B, Hermsmeyer J, Norvell DC (2010) Chronic sacroiliac joint pain: fusion versus denervation as treatment options. EBSJ; 1(3):35-44.

2. Dreyfuss P, Dreyer SJ, Cole A, et al (2004) Sacroiliac joint pain. J Am Acad Orthop Surg; 12(4):255-265. 